\title{
HCI evaluation with Cognitive Dimensions of Notations
}

\author{
Juliana Jansen Ferreira, Vinícius Segura \\ IBM Research \\ Rio de Janeiro, Brazil \\ \{jjansen,vboas\}@br.ibm.com
}

A practical course using the Cognitive Dimensions of Notations framework

\begin{abstract}
During the design and development of a technological artifact or when such artifact is already in the hands of users, an inspection method can help researchers and practitioners deal with not only usability issues, but also provide a common language to evaluate different design alternatives. The Cognitive Dimensions of Notations framework (CDNf) is a methodological resource designed to identify and discuss cognitive loads imposed by notations. In this six-hour short course, we aim to talk about our experience with CDNf - alone and combined with other HCI methods - and to explore CDNf in practice - with hands-on activities to show participants how it can be a useful resource.
\end{abstract}

\section{KEYWORDS}

Evaluation methods, inspection methods, Cognitive Dimensions of Notation, notations, technological artifacts.

\section{Motivation}

Inspection methods are important resources for HumanComputer Interaction (HCI) researchers and practitioners. One of the reasons is that inspection methods are lower-cost evaluation methods - expert-based - than user observation ones. The other reason is that inspection methods can frame evidence collected during inspections in such a way that the evaluator can more easily establish certain relations among various instances and kinds of data $[4]([8]$, pp. 269). During the design and development of a technological artifact - its formative phase or when the artifact is in the hands of users - its summative phase - an inspection method can help researchers and practitioners to deal with more than just usability issues ([8], pp. 271-272). Especially in the design phase, when the notations to communicate with users are being defined by designers and developers, a resource to discuss these new notations can be helpful to identify early problems that can be propagated and cause issues in the technology artifact interaction.

Permission to reproduce or distribute, in whole or in part, material extracted from this work, verbatim, adapted or remixed, as well as the creation or production from the content of such work, is granted without fee for non-commercial use, provided that the original work is properly credited.

IHC 2019 - MINICURSOS, Outubro 21-25, 2019, Vitória, Brasil. In Anais Estendidos do XVIII Simpósio Brasileiro sobre Fatores Humanos em Sistemas Computacionais. Porto Alegre: SBC.

(c) 2019 by the author(s), in accordance with the terms of the Creative Commons Attribution-NonCommercial 4.0 International Public License (CC BY-NC 4.0).
Table 1. The list of Cognitive Dimensions of Notations CDN (2003)

\begin{tabular}{l|l}
\hline Cognitive dimension & Description \\
\hline Abstraction & Types and availability of abstraction mechanisms \\
\hline Closeness of mapping & Closeness of representation to domain \\
\hline Consistency & $\begin{array}{l}\text { Similar semantics are expressed in similar syntactic } \\
\text { forms }\end{array}$ \\
\hline Diffuseness & Verbosity of language \\
\hline Error-proneness & $\begin{array}{l}\text { The notation invites mistakes and the system gives little } \\
\text { protection }\end{array}$ \\
\hline Hard mental operations & High demand on cognitive resources \\
\hline Hidden dependencies & Relevant relations between entities are not visible \\
\hline Premature commitment & Constraints on the order of doing things \\
\hline Progressive evaluation & Work-to-date can be checked at any time \\
\hline Provisionality & Degree of commitment to actions or marks \\
\hline Role-expressiveness & The purpose of an entity is readily inferred \\
\hline Secondary notation & Extra information in means other than formal syntax \\
\hline Viscosity & Resistance to change \\
\hline Visibility & Ability to view entities easily \\
\hline
\end{tabular}

The Cognitive Dimensions of Notations framework (CDNf) is a prime candidate for evaluating artifacts dealing with notations. It was proposed to evaluate the usability of information artifacts ${ }^{1}$. It defines a set of design principles (Table 1) for creating or evaluating notations, user interfaces, and programming languages used with information artifacts. This framework provides a common vocabulary for discussing many cognitive factors of such representation-building systems. It aims to improve the quality of discussions and decisions in design and evaluation activity [1].

Although the application of CDNf for usability inspection have been discussed for more than twenty years ago [6][7], its motivation topics are still relevant, particularly as a $\mathrm{HCI}$ evaluation method: CDNf (a) offers a comprehensible, broadbrush evaluation, (b) uses terms that were readily comprehended by non-specialists, (c) is theoretically coherent, and, especially, (d) distinguishes between the needs of different types of user needs [1]. CDNf as a discussion tool is particularly useful since the cognitive dimensions describe generic aspects of usability, rather than features of the system under study, and leaves the user free to comment on aspects of the system that the designer may not have anticipated [1].

We have a large experience with inspection methods, especially the CDNf. Throughout Juliana's Ph.D. research and thesis [3], since 2011, we have been investigating HCI inspection methods.

${ }^{1}$ https://www.cl.cam.ac.uk/ afb21/CognitiveDimensions/index.html 
We have investigated the CDNf application as an isolated method, to discuss specific types of notation: for investigating visualization and insights [2] and for inspecting history visualization interfaces [9]. We also have investigated and evaluated its application combined with other methods, particularly the Semiotic Inspection Method from the Semiotic Engineering theory [3][4][5][10].

\section{Extended Summary}

This six-hours short course will be structure as follows:

(1) Participants' profile survey (online, before course)- get to know participants' experience and interest on inspection methods.

(2) Introduction and presentation $(\sim 0: 30)$.

(3) HCI Evaluation Methods ( 0:30) - overview of HCI evaluation methods, focusing on inspection methods like CDNf.

(4) CDNf Overview ( 1:00) - explanation about CDNf theory basis, the cognitive dimensions, and their use.

(5) CDNf practice cases ( 3:30)- dynamic activities to exercise using $\mathrm{CDNf}$ in different scenarios.

(6) Wrap-up ( 0:30)- discuss the course results, participants' feedback, final questions, and comments.

\section{Audience}

This short course targets an audience of enthusiasts in HCI evaluation methods. Students can have a hands-on experience collaborating with experts and more experienced researchers can learn more about CDNf. We aim to provide a practical learning environment, where we will present real-case examples, but will also encourage participants to bring their own contexts of interest.

\section{Authors' biographies}

Juliana Jansen Ferreira is a User eXperience researcher at the Visual Analytics \& Comprehension Research group of IBM Research Brazil. She holds a D.Sc. degree in Human-Computer Interaction from the Pontifical Catholic University of Rio de Janeiro (PUC-Rio). In her thesis and related book [10], she developed an inspection method called SigniFYI Models, which combines communicative and cognitive perspectives, taking into consideration the tools, notations, and people who work with models throughout the software engineering process. The cognitive perspective of SigniFYI Models is based on the CDNf. Skilled and thorough qualitative researcher, Juliana is used to analyze large amount of data to point issues and insights for further investigations related to people and how technology can influence their lives. Her work has been published in worldleading venues such as ICEIS, VL/HCC, CHI, HCII, and ETRA and she is also co-author in a Springer Book entitled "Software Developers as Users".

Vinícius Segura is a Research Staff Member at IBM Research in Rio de Janeiro, working in the Visual Analytics \& Comprehension group. He holds a D.Sc. and M.Sc. degree in Human-Computer Interaction and a B.Sc. in Computer Engineering, both from PUC-Rio. Over the last years, he has worked in several computer graphics and information visualization projects. His work has been published in venues such as VL/HCC, EICS, AVI and CHI.

\section{Additional information and details}

The course will be held in Portuguese, but all the material provided will be in English like any produced artifacts during the course's dynamics. We will need the following resource for this course: projector with HDMI connection, post-its, flipcharts and whiteboard.

\section{REFERENCES}

[1] Alan Blackwell and Thomas Green. 2003. Notational systems-the cognitive dimensions of notations framework. HCI Models, Theories, and Frameworks: Toward an Interdisciplinary Science. Morgan Kaufmann.

[2] J. Ferreira, V. Segura, and R. Cerqueira. 2015. Cognitive Dimensions of Notation tailored to environments for visualization and insights.

[3] Juliana J Ferreira. 2015. Comunicação através de modelos no contexto do desenvolvimento de Software. PUC-Rio.

[4] Juliana Jansen Ferreira, Clarisse Sieckenius de Souza, and Renato Cerqueira 2015. Why and how to investigate interaction design of software development tools. SBC Journal on Interactive Systems 6, 1: 48-65.

[5] Juliana Soares Jansen Ferreira, Clarisse Sieckenius de Souza, Rafael Rossi de Mello Brandão, and Carla Faria Leitão. 2018. SigniFYI-CDN: merged communicability and usability methods to evaluate notation-intensive interaction. arXiv preprint arXiv:1808.08138.

[6] Thomas Green and Alan Blackwell. 1998. Cognitive dimensions of information artefacts: a tutorial. In BCS HCI Conference.

[7] Thomas R. G. Green and Marian Petre. 1996. Usability analysis of visual programming environments: a 'cognitive dimensions' framework. Journal of Visual Languages \& Computing 7, 2: 131-174.

[8] Jonathan Lazar, Jinjuan Heidi Feng, and Harry Hochheiser. 2017. Research methods in human-computer interaction. Morgan Kaufmann.

[9] Vinícius Segura, Juliana Jansen Ferreira, Renato Cerqueira, and Simone Diniz Junqueira Barbosa. 2016. An Analytical Evaluation of a User Interaction History Visualization System Using CDN and PoN. In Proceedings of the 15th Brazilian Symposium on Human Factors in Computing Systems (IHC '16).

[10] Clarisse Sieckenius de Souza, Renato Fontoura de Gusmão Cerqueira, Luiz Marques Afonso, Rafael Rossi de Mello Brandão, and Juliana Soares Jansen Ferreira. 2016. Software Developers as Users. Springer International Publishing, Cham. 